\title{
Atiyah Classes and Closed Forms on Moduli Spaces of Sheaves
}

\author{
Francesco BotTacin (*)
}

ABstRACT - Let $X$ be a smooth $n$-dimensional projective variety, and let $Y$ be a moduli space of stable sheaves on $X$. By using the local Atiyah class of a universal family of sheaves on $Y$, which is well defined even when such a universal family does not exist, we are able to construct natural maps

$$
f: H^{i}\left(X, \Omega_{X}^{j}\right) \longrightarrow H^{k+i-n}\left(Y, \Omega_{Y}^{k+j-n}\right),
$$

for any $i, j=1, \ldots, n$ and any $k \geq \max \{n-i, n-j\}$. In particular, for $k=n-i$, the map $f$ associates a closed differential form of degree $j-i$ on the moduli space $Y$ to any element of $H^{i}\left(X, \Omega_{X}^{j}\right)$. This method provides a natural way to construct closed differential forms on moduli spaces of sheaves. We remark that no smoothness hypothesis is made on the moduli space $Y$. As an application, we describe the construction of closed differential forms on the Hilbert schemes of points of $X$.

\section{Introduction.}

Let $X$ be a smooth projective variety, defined over an algebraically closed field $k$ of characteristic 0 , and let $Y$ be a moduli space of stable sheaves on $X$. Usually $Y$ will be a singular quasi-projective variety, whose points parametrize isomorphism classes of stable coherent sheaves of $\mathcal{O}_{X^{-}}$ modules with a fixed Hilbert polynomial.

It is by now well known that many geometric properties of the moduli space $Y$ are determined by similar properties of the variety $X$. One of the first examples was discovered by S. Mukai in [Mu1], where he proved that

(*) Indirizzo dell'A.: Dipartimento di Matematica Pura e Applicata, Università di Padova, Via Trieste, 63 - 35121 Padova.

E-mail: bottacin@math.unipd.it

2000 Mathematics Subject Classification. Primary: 14J60, Secondary: 14D20, $58 \mathrm{~A} 10$. 
if $X$ is a symplectic algebraic surface (i.e., an abelian or K3 surface), one can construct, in a natural way, a non-degenerate 2 -form on the moduli space $Y$ of stable sheaves on $X$. The closedness of this 2 -form was later proved by the same author in [Mu2], but only for stable vector bundles. A proof of the closedness of Mukai's 2-form valid in general was finally given in [HL].

Mukai's result was later generalized by S. Kobayashi [K] to the case of the moduli space of simple vector bundles over a compact Kähler manifold $X$ endowed with a holomorphic symplectic structure.

A different proof of the closedness of these so-called "trace forms" can be found in $[R]$. In this case too, the proof is given only for locally free sheaves.

Tyurin [Ty] gave another example of the relations between geometric properties of the moduli space $Y$ and those of the space $X$, by proving that the choice of a Poisson structure on an algebraic Poisson surface $X$ determines a Poisson structure on the moduli space $Y$ of stable vector bundles on $X$. Actually Tyurin did not prove that the Poisson bracket constructed on $Y$ satisfies the Jacobi identity; this is nevertheless true, as we proved later in [B1].

In the present paper we shall prove the following result:

Theorem. Let $n=\operatorname{dim} X$. For any $i, j=1, \ldots, n$ and any $k \geq$ $\geq \max \{n-i, n-j\}$, there is a natural map

$$
f: H^{i}\left(X, \Omega_{X}^{j}\right) \longrightarrow H^{k+i-n}\left(Y, \Omega_{Y}^{k+j-n}\right) .
$$

Moreover, for any $\sigma \in H^{i}\left(X, \Omega_{X}^{j}\right)$, the element $f(\sigma)$ is d-closed.

As a corollary, we obtain, for $k=n-i$ and any $i \leq j$, a natural map

$$
f: H^{i}\left(X, \Omega_{X}^{j}\right) \longrightarrow H^{0}\left(Y, \Omega_{Y}^{j-i}\right),
$$

i.e., we can construct closed differential forms of degree $j-i$ on the moduli space $Y$ starting from elements in $H^{i}\left(X, \Omega_{X}^{j}\right)$. In particular, if $X$ has trivial canonical bundle, we obtain a (natural) closed $n$-form $f(1) \in H^{0}\left(Y, \Omega_{Y}^{n}\right)$ on any moduli space of stable sheaves on $X$. This result may be considered as a higher dimensional generalization of Mukai's result.

After a first version of this paper was completed, we came across the paper [KM], where the authors construct closed 2-forms on the smooth locus of some moduli spaces of sheaves via the Atiyah class of a universal family, using a construction that is essentially a specialization of our construction of closed $n$-forms to the case $n=2$. In some cases they are also 
able to prove that the resulting 2-forms are non-degenerate, hence they define a symplectic structure on the moduli space $Y$.

However, the results concerning the existence of closed holomorphic 2forms contained in $[\mathrm{KM}]$ are stated and proved only for the smooth locus of the moduli spaces of stable sheaves. This is, in general, a very strong limitation; in fact, moduli spaces of stable sheaves on an algebraic variety $X$ of dimension greater than 2 are almost always singular. Moreover, the use of the Atiyah class of a universal family of sheaves is problematic, since usually there does not exist such a universal family of sheaves. As we shall prove in the sequel, these difficulties can be overcome by using a local version of the Atiyah class of some (locally defined) universal family of sheaves.

Let us briefly describe now the organization of the paper. In Section 1 we start by recalling some basic results about cup-products and trace maps for coherent sheaves. Then we recall the definition of the Atiyah class of a coherent sheaf and we introduce the notion of the local Atiyah class of a flat family of coherent sheaves. In a relative setting, the local Atiyah class turns out to be the natural object to consider. In fact, we shall prove that, when we consider a moduli space $Y$ of stable sheaves on a smooth projective variety $X$, the local Atiyah class of a universal family of sheaves on $Y$ is well defined even when such a universal family does not exist (which is usually the case).

Finally, we introduce some cohomology classes, called Newton polynomials, constructed by taking the trace of repeated compositions of the Atiyah class with itself. In this case too, in a relative setting, there exists a local version of the Newton polynomials, constructed by using the local Atiyah class.

In Section 2 we use the local Newton polynomials of a universal family of sheaves to define the map

$$
f: H^{i}\left(X, \Omega_{X}^{j}\right) \longrightarrow H^{k+i-n}\left(Y, \Omega_{Y}^{k+j-n}\right) .
$$

Then we prove that the elements in the image of $f$ are $d$-closed.

As an application, we show how these results can be applied in order to construct closed differential forms on Hilbert schemes of points of a smooth projective variety.

\section{Preliminaries.}

\section{1 - Cup-product and trace maps.}

In this section we shall briefly recall some standard results about trace maps and cup-products. For more details (and proofs) we refer to [A] or [HL]. 
Let $X$ be a scheme of finite type over a field $k$ of characteristic 0 . In the sequel, whenever we speak of a sheaf on $X$ we shall always mean a sheaf of $\mathcal{O}_{X}$-modules. Let $E$ be a locally free sheaf of finite rank on $X$. The usual trace map

$$
\operatorname{tr}: \mathcal{E} n d(E) \rightarrow \mathcal{O}_{X}
$$

induces natural maps

$$
\operatorname{tr}: \operatorname{Ext}^{i}(E, E) \rightarrow H^{i}\left(X, \mathcal{O}_{X}\right),
$$

for any $i \geq 0$. More generally, for any coherent sheaf $\mathcal{F}$ on $X$, we can define a trace map

$$
\operatorname{tr}: \mathcal{H o m}(E, E \otimes \mathcal{F}) \rightarrow \mathcal{F} .
$$

In this case the induced maps on cohomology are

$$
\operatorname{tr}: \operatorname{Ext}^{i}(E, E \otimes \mathcal{F}) \rightarrow H^{i}(X, \mathcal{F}) .
$$

If $E, F$ and $G$ are three locally free sheaves on $X$, there is a cup-product (or Yoneda composition) map

$$
\operatorname{Ext}^{i}(F, G) \times \operatorname{Ext}^{j}(E, F) \stackrel{\circ}{\longrightarrow} \operatorname{Ext}^{i+j}(E, G) .
$$

In the special case $E=F=G$, we find that the composition of the cupproduct and trace maps is graded commutative in the following sense: if $\alpha \in \operatorname{Ext}^{i}(E, E)$ and $\beta \in \operatorname{Ext}^{j}(E, E)$, then

$$
\operatorname{tr}(\alpha \circ \beta)=(-1)^{i j} \operatorname{tr}(\beta \circ \alpha)
$$

as an element of $H^{i+j}\left(X, \mathcal{O}_{X}\right)$.

Analogous maps can be easily defined when the locally free sheaves $E$, $F$ and $G$ are replaced by finite complexes $E, F$ and $G$ of locally free sheaves of finite rank on $X$. This fact can be used to define cup-products and trace maps also for coherent sheaves $\mathcal{E}, \mathcal{F}$ and $\mathcal{G}$ on $X$, provided that these sheaves admit finite resolutions by locally free sheaves. This is the case, for instance, if $X$ is a smooth projective variety.

There is also a relative version of the preceding constructions.

Let $X$ and $Y$ be two schemes of finite type over $k$ and $\mathcal{E}, \mathcal{F}$ and $\mathcal{G}$ be $Y$ flat families of coherent sheaves on $X$, i.e., coherent sheaves on $X \times Y$, flat over $Y$. We shall always assume that all these sheaves admit finite locally free resolutions. We shall denote by $p: X \times Y \rightarrow X$ and $q: X \times Y \rightarrow Y$ the canonical projections and by $\mathcal{E} x t_{q}^{i}(\mathcal{E}, \mathcal{F})$ the $i$-th relative Ext-sheaf, i.e., the $i$-th derived funtor of $q_{*} \mathcal{H}_{0 m_{\mathcal{O}_{X \times Y}}}(\mathcal{E}, \mathcal{F})$. 
In this situation there is a relative trace map

$$
\operatorname{tr}: \mathcal{E} x t_{q}^{i}(\mathcal{E}, \mathcal{E} \otimes \mathcal{F}) \rightarrow R^{i} q_{*} \mathcal{F}
$$

and a relative cup-product

$$
\mathcal{E}_{x} t_{q}^{i}(\mathcal{F}, \mathcal{G}) \times \mathcal{E}_{x} t_{q}^{j}(\mathcal{E}, \mathcal{F}) \stackrel{\circ}{\longrightarrow} \mathcal{E} x t_{q}^{i+j}(\mathcal{E}, \mathcal{G}),
$$

satisfying the analogue of (1.1) when $\mathcal{E}=\mathcal{F}=\mathcal{G}$.

\section{2 - The Atiyah class.}

Let $X$ be a scheme of finite type over $k$ and $\mathcal{E}$ be a coherent sheaf on $X$ which admits a finite locally free resolution. Let us denote by $\mathcal{P}_{X}^{1}(\mathcal{E})$ the sheaf of principal parts of order 1 of sections of $\mathcal{E}$ (cf. [EGA4, 4]). There is a natural exact sequence

$$
0 \rightarrow \mathcal{E} \otimes \Omega_{X}^{1} \rightarrow \mathcal{P}_{X}^{1}(\mathcal{E}) \rightarrow \mathcal{E} \rightarrow 0
$$

hence $\mathcal{P}_{X}^{1}(\mathcal{E})$ can be regarded as an extension of $\mathcal{E}$ by $\mathcal{E} \otimes \Omega_{X}^{1}$.

Definition 1.1. The Atiyah class of $\mathcal{E}$ is the class

$$
a(\mathcal{E}) \in \operatorname{Ext}^{1}\left(\mathcal{E}, \mathcal{E} \otimes \Omega_{X}^{1}\right)
$$

corresponding to the extension (1.2).

By taking the trace, we get an element $\operatorname{tr}(a(\mathcal{E})) \in H^{1}\left(X, \Omega_{X}^{1}\right)$ : this is just the opposite of the first Chern class $c_{1}(\mathcal{E})$.

If $Y$ is another scheme of finite type over $k$ and $\mathcal{E}$ is a $Y$-flat family of coherent sheaves on $X$, admitting a finite locally free resolution, we have a global Atiyah class

$$
a(\mathcal{E}) \in \operatorname{Ext}_{X \times Y}^{1}\left(\mathcal{E}, \mathcal{E} \otimes \Omega_{X \times Y}^{1}\right),
$$

defined as above. However, in this relative situation we are more interested in a "local" version of the Atiyah class of $\mathcal{E}$, which we shall denote by $\tilde{a}(\mathcal{E})$.

Definition 1.2. The local Atiyah class of $\mathcal{E}$ is the section

$$
\tilde{a}(\mathcal{E}) \in H^{0}\left(Y, \mathcal{E} x t_{q}^{1}\left(\mathcal{E}, \mathcal{E} \otimes \Omega_{X \times Y}^{1}\right)\right)
$$


defined as the image of $a(\mathcal{E})$ under the natural map

$$
\operatorname{Ext}_{X \times Y}^{1}\left(\mathcal{E}, \mathcal{E} \otimes \Omega_{X \times Y}^{1}\right) \rightarrow H^{0}\left(Y, \mathcal{E} x t_{q}^{1}\left(\mathcal{E}, \mathcal{E} \otimes \Omega_{X \times Y}^{1}\right)\right)
$$

coming from the local-to-global spectral sequence $H^{i}\left(Y, \mathcal{E} x t_{q}^{j}\right) \Rightarrow \operatorname{Ext}_{X \times Y}^{i+j}$

We shall now explain why, in a relative setting, the "correct" object to consider is not the global Atiyah class $a(\mathcal{E})$ but the local one $\tilde{a}(\mathcal{E})$.

First of all, let us recall that two $Y$-flat families $\mathcal{E}$ and $\mathcal{F}$ of coherent sheaves on $X$ are said to be equivalent if $\mathcal{F} \cong \mathcal{E} \otimes_{\mathcal{O}_{X \times Y}} q^{*} L$, for some invertible sheaf $L$ on $Y$. Since the Atiyah class of a tensor product of sheaves is given by

$$
a\left(\mathcal{E}_{1} \otimes \mathcal{E}_{2}\right)=a\left(\mathcal{E}_{1}\right) \otimes \mathrm{id}_{\mathcal{E}_{2}}+\mathrm{id}_{\mathcal{E}_{1}} \otimes a\left(\mathcal{E}_{2}\right),
$$

it follows that $a(\mathcal{F})=a\left(\mathcal{E} \otimes_{\mathcal{O}_{X \times Y}} q^{*} L\right) \neq a(\mathcal{E})$, in general. However, if we consider the local Atiyah classes, we have:

Lemma 1.3. Let $\mathcal{E}$ and $\mathcal{F}$ be two $Y$-flat families of coherent sheaves on $X$. If $\mathcal{E}$ and $\mathcal{F}$ are equivalent, then the local Atiyah classes $\tilde{a}(\mathcal{E})$ and $\tilde{a}(\mathcal{F})$ are identified under the natural isomorphism

$$
H^{0}\left(Y, \mathcal{E} x t_{q}^{1}\left(\mathcal{E}, \mathcal{E} \otimes \Omega_{X \times Y}^{1}\right)\right) \cong H^{0}\left(Y, \mathcal{E} x t_{q}^{1}\left(\mathcal{F}, \mathcal{F} \otimes \Omega_{X \times Y}^{1}\right)\right) .
$$

Proof. If $\mathcal{E}$ and $\mathcal{F}$ are equivalent we can write $\mathcal{F} \cong \mathcal{E} \otimes q^{*} L$, for some invertible sheaf $L$ on $Y$. Since $L$ is locally free of rank one, there is a natural isomorphism of sheaves

$$
\mathcal{E} x t_{q}^{1}\left(\mathcal{E}, \mathcal{E} \otimes \Omega_{X \times Y}^{1}\right) \cong \mathcal{E} x t_{q}^{1}\left(\mathcal{F}, \mathcal{F} \otimes \Omega_{X \times Y}^{1}\right),
$$

hence, from now on, we shall identify these two sheaves.

By taking the global Atiyah class of $\mathcal{F}$, we have

$$
a(\mathcal{F})=a(\mathcal{E}) \otimes \operatorname{id}_{q^{*} L}+\operatorname{id}_{\mathcal{E}} \otimes a\left(q^{*} L\right) .
$$

The difference between $a(\mathcal{F})$ and $a(\mathcal{E})$, under the natural identification (1.4) induced by (1.5), is then given by $\mathrm{id}_{\mathcal{E}} \otimes a\left(q^{*} L\right)=\mathrm{id}_{\mathcal{E}} \otimes q^{*}(a(L))$, where

$$
a(L) \in \operatorname{Ext}_{Y}^{1}\left(L, L \otimes \Omega_{Y}^{1}\right) \cong H^{1}\left(Y, \Omega_{Y}^{1}\right) .
$$

The standard exact sequence associated to the local-to-global spectral sequence for the Ext groups is

$H^{1}\left(Y, q_{*} \mathcal{H o m}\left(\mathcal{E}, \mathcal{E} \otimes \Omega_{X \times Y}^{1}\right)\right) \rightarrow \operatorname{Ext}^{1}\left(\mathcal{E}, \mathcal{E} \otimes \Omega_{X \times Y}^{1}\right) \rightarrow H^{0}\left(Y, \mathcal{E} x t_{q}^{1}\left(\mathcal{E}, \mathcal{E} \otimes \Omega_{X \times Y}^{1}\right)\right)$. 
Now it can be checked that $\operatorname{id}_{\mathcal{E}} \otimes a\left(q^{*} L\right)$ belongs to $H^{1}\left(Y, q_{*} \mathcal{H o m}(\mathcal{E}, \mathcal{E} \otimes\right.$ $\left.\left.\otimes \Omega_{X \times Y}^{1}\right)\right)$, hence its image in $H^{0}\left(Y, \mathcal{E} x t_{q}^{1}\left(\mathcal{E}, \mathcal{E} \otimes \Omega_{X \times Y}^{1}\right)\right)$ is zero. It follows that $\tilde{a}(\mathcal{F})=\tilde{a}(\mathcal{E})$ as stated.

\section{3 - The Newton polynomials.}

Let $X$ be a scheme of finite type over $k$ and $\mathcal{E}$ be a coherent sheaf on $X$ that admits a finite resolution by locally free sheaves. By repeatedly composing the Atiyah class $a(\mathcal{E}) \in \operatorname{Ext}^{1}\left(\mathcal{E}, \mathcal{E} \otimes \Omega_{X}^{1}\right)$ with itself, we obtain classes in $\operatorname{Ext}^{i}\left(\mathcal{E}, \mathcal{E} \otimes\left(\Omega_{X}^{1}\right)^{\otimes i}\right)$, for any $i \geq 1$. Then, by composing with the map induced by the natural map $\left(\Omega_{X}^{1}\right)^{\otimes i} \rightarrow \Omega_{X}^{i}$, we get classes $a(\mathcal{E})^{i} \in$ $\in \operatorname{Ext}^{i}\left(\mathcal{E}, \mathcal{E} \otimes \Omega_{X}^{i}\right)$.

We now give the following definition:

Definition 1.4. The $i$-th Newton polynomial of $\mathcal{E}$ is the cohomology class

$$
\gamma^{i}(\mathcal{E})=\operatorname{tr}\left(a(\mathcal{E})^{i}\right) \in H^{i}\left(X, \Omega_{X}^{i}\right)
$$

The Newton polynomials of $\mathcal{E}$ coincide, up to a scalar factor, with the components of the Chern character of $\mathcal{E}$ and, as the Chern classes, they are d-closed. More precisely:

LEMMA 1.5. Let $d: H^{i}\left(X, \Omega_{X}^{i}\right) \rightarrow H^{i}\left(X, \Omega_{X}^{i+1}\right)$ be the map induced by the exterior differential $d: \Omega_{X}^{i} \rightarrow \Omega_{X}^{i+1}$. Then $d \gamma^{i}(\mathcal{E})=0$.

For the proof of this result we refer the reader to [HL, Sect. 10.1.6].

In a relative situation, where $\mathcal{E}$ is a $Y$-flat family of coherent sheaves on $X$, we have the global Newton polynomials $\gamma^{i}(\mathcal{E}) \in H^{i}\left(X \times Y, \Omega_{X \times Y}^{i}\right)$ and also their local versions, constructed by using the local Atiyah class of $\mathcal{E}$,

$$
\tilde{\gamma}^{i}(\mathcal{E}) \in H^{0}\left(Y, R^{i} q_{*}\left(\Omega_{X \times Y}^{i}\right)\right) .
$$

We note that $\tilde{\gamma}^{i}(\mathcal{E})$ is the image of $\gamma^{i}(\mathcal{E})$ under the natural map

$$
H^{i}\left(X \times Y, \Omega_{X \times Y}^{i}\right) \rightarrow H^{0}\left(Y, R^{i} q_{*}\left(\Omega_{X \times Y}^{i}\right)\right)
$$

sending a global section of a presheaf to a global section of the associated sheaf.

Exactly as for the global Newton polynomials, the local ones too are $d$ closed, i.e., we have $d \tilde{\gamma}^{i}(\mathcal{E})=0$. 


\section{Closed Forms on Moduli Spaces.}

From now on we shall denote by $X$ a smooth projective variety defined over $k$ and we take as $Y$ a moduli space of stable sheaves on $X$.

For the definition and the construction of moduli spaces of stable sheaves on $X$ we refer to [S]: what we need to know, for the moment, is that moduli spaces of stable sheaves exist and they are quasi-projective varieties (usually singular).

In order to apply the constructions described in the previous section we need to know that finite locally free resolutions of coherent sheaves exist: this is indeed the case since $X$ is a smooth projective variety.

Finally, there is another technical problem we have to deal with: the nonexistence of universal families of sheaves. In general, on a moduli space $Y$ of stable sheaves on $X$ there does not exist a universal family of sheaves $\mathcal{E}$, not even locally for the Zariski topology. However, universal families on $Y$ exist locally in the étale topology (or in the usual complex topology, if the base field $k$ is the complex field) [S, Theorem 1.21]. These local universal families are not uniquely determined, they are defined only up to tensoring with the pullback of an invertible sheaf on $Y$. Usually these ambiguities prevent the local universal families to glue together to a globally defined one.

Let us denote by $\mathcal{E}_{\alpha}$ a collection of local universal families, one for each open set $U_{\alpha}$ of a suitable covering of the moduli space $Y$ (in the appropriate topology). Even if the sheaves $\mathcal{E}_{\alpha}$ do not glue to define a global universal family $\mathcal{E}$, the relative Ext-sheaves $\mathcal{E} x t_{q}^{i}\left(\mathcal{E}_{\alpha}, \mathcal{E}_{\alpha}\right)$ and $\mathcal{E} x t_{q}^{i}\left(\mathcal{E}_{\alpha},\left.\mathcal{E}_{\alpha} \otimes \Omega_{X \times Y}^{i}\right|_{X \times U_{\alpha}}\right)$ do indeed glue to define global sheaves on $Y$. For this reason, by abusing notation, we shall denote these sheaves by $\mathcal{E} x t_{q}^{i}(\mathcal{E}, \mathcal{E})$ and $\mathcal{E} x t_{q}^{i}\left(\mathcal{E}, \mathcal{E} \otimes \Omega_{X \times Y}^{i}\right)$, even if the universal family $\mathcal{E}$ does not exist globally on $Y$.

Since each $\mathcal{E}_{\alpha}$ is defined only up to tensoring with the pull-back of an invertible sheaf on $U_{\alpha}$, the global Atiyah class $a\left(\mathcal{E}_{\alpha}\right)$ is not well defined. However, by recalling the result of Lemma 1.3, we see that the local Atiyah class

$$
\tilde{a}\left(\mathcal{E}_{\alpha}\right) \in H^{0}\left(U_{\alpha}, \mathcal{E} x t_{q}^{1}\left(\mathcal{E}_{\alpha},\left.\mathcal{E}_{\alpha} \otimes \Omega_{X \times Y}^{1}\right|_{X \times U_{\alpha}}\right)\right)=H^{0}\left(U_{\alpha}, \mathcal{E} x t_{q}^{1}\left(\mathcal{E}, \mathcal{E} \otimes \Omega_{X \times Y}^{1}\right)\right)
$$

is well defined, since it depends only on the equivalence class of $\mathcal{E}_{\alpha}$. Now it is easy to check that all these local sections $\tilde{a}\left(\mathcal{E}_{\alpha}\right)$ glue together to define a global section of the sheaf $\mathcal{E} x t_{q}^{1}\left(\mathcal{E}, \mathcal{E} \otimes \Omega_{X \times Y}^{1}\right)$. Again, by abuse of notation, we shall denote this global section by

$$
\tilde{a}(\mathcal{E}) \in H^{0}\left(Y, \mathcal{E} x t_{q}^{1}\left(\mathcal{E}, \mathcal{E} \otimes \Omega_{X \times Y}^{1}\right)\right),
$$

even if the universal family $\mathcal{E}$ does not exist. 
Analogous considerations hold also for the Newton polynomials: while the global Newton polynomials $\gamma^{i}(\mathcal{E})$ are not defined, there are well defined local Newton polynomials

$$
\tilde{\gamma}^{i}(\mathcal{E}) \in H^{0}\left(Y, R^{i} q_{*}\left(\Omega_{X \times Y}^{i}\right)\right) .
$$

Now, for any open subset $U \subseteq Y$ we have a direct sum decomposition $\Omega_{X \times U}^{1}=p^{*} \Omega_{X}^{1} \oplus q^{*} \Omega_{U}^{1}$. Since $X$ is a smooth variety, it follows that there is a Künneth decomposition

$$
H^{n}\left(X \times U, \Omega_{X \times U}^{n}\right)=\bigoplus_{i, j=0}^{n} H^{i}\left(X, \Omega_{X}^{j}\right) \otimes H^{n-i}\left(U, \Omega_{U}^{n-j}\right),
$$

for every $n \geq 0$. The sheaf on $Y$ associated to the presheaf

$$
U \mapsto H^{n}\left(X \times U, \Omega_{X \times U}^{n}\right)
$$

is the higher direct image $R^{n} q_{*}\left(\Omega_{X \times Y}^{n}\right)$ and, from the above Künneth decomposition, we obtain an analogous decomposition of sheaves

$$
R^{n} q_{*}\left(\Omega_{X \times Y}^{n}\right)=\bigoplus_{i, j=0}^{n} H^{i}\left(X, \Omega_{X}^{j}\right) \otimes \mathcal{H}^{n-i}\left(Y, \Omega_{Y}^{n-j}\right),
$$

where $\mathcal{H}^{n-i}\left(Y, \Omega_{Y}^{n-j}\right)$ is the sheaf associated to the presheaf

$$
U \mapsto H^{n-i}\left(U, \Omega_{U}^{n-j}\right)
$$

and where, by abuse of notation, we have written $H^{i}\left(X, \Omega_{X}^{j}\right)$ to denote the corresponding constant sheaf on $Y$.

By using this direct sum decomposition, we obtain a decomposition of the local Newton polynomials:

Definition 2.1. For a Newton polynomial $\tilde{\gamma}^{n}(\mathcal{E}) \in H^{0}\left(Y, R^{n} q_{*}\left(\Omega_{X \times Y}^{n}\right)\right)$, we shall write $\tilde{\gamma}^{n}(\mathcal{E})=\sum_{i, j} \tilde{\gamma}_{i, j}^{n}(\mathcal{E})$, where $\tilde{\gamma}_{i, j}^{n}(\mathcal{E})$ is a global section of the sheaf $H^{i}\left(X, \Omega_{X}^{j}\right) \otimes \mathcal{H}^{n-i}\left(Y, \Omega_{Y}^{n-j}\right)$.

We note that the restriction of $\tilde{\gamma}_{i, j}^{n}(\mathcal{E})$ to a sufficiently small open subset $U \subseteq Y$ gives a section

$$
\left.\tilde{\gamma}_{i, j}^{n}(\mathcal{E})\right|_{U} \in H^{i}\left(X, \Omega_{X}^{j}\right) \otimes H^{n-i}\left(U,\left.\Omega_{Y}^{n-j}\right|_{U}\right) .
$$

We are now able to prove the following result: 
Theorem 2.2. Let $n=\operatorname{dim} X$. For any $i, j=1, \ldots, n$ and any $k \geq \max \{n-i, n-j\}$, there is a natural map

$$
f: H^{i}\left(X, \Omega_{X}^{j}\right) \longrightarrow H^{k+i-n}\left(Y, \Omega_{Y}^{k+j-n}\right) .
$$

Moreover, for any $\sigma \in H^{i}\left(X, \Omega_{X}^{j}\right)$, the element $f(\sigma)$ is d-closed.

Proof. The map $f$ is defined by composing the isomorphism

$$
H^{i}\left(X, \Omega_{X}^{j}\right) \stackrel{\sim}{\longrightarrow} H^{n-i}\left(X, \Omega_{X}^{n-j}\right)^{*}
$$

given by Serre duality, with the map

$$
H^{n-i}\left(X, \Omega_{X}^{n-j}\right)^{*} \longrightarrow H^{k+i-n}\left(Y, \Omega_{Y}^{k+j-n}\right)
$$

induced by multiplication by the section $\tilde{\gamma}_{n-i, n-j}^{k}(\mathcal{E})$ of the sheaf $H^{n-i}\left(X, \Omega_{X}^{n-j}\right) \otimes \mathcal{H}^{k+i-n}\left(Y, \Omega_{Y}^{k+j-n}\right)$. It only remains to show that, for any $\sigma \in H^{i}\left(X, \Omega_{X}^{j}\right)$, we have $d(f(\sigma))=0$. The proof is an adaptation of the one given in [HL] for 2-forms.

Let us write $\tilde{\gamma}_{n-i, n-j}^{k}(\mathcal{E})=\sum_{l} \alpha_{l} \otimes \beta_{l}$, for some $\alpha_{l} \in H^{n-i}\left(X, \Omega_{X}^{n-j}\right)$ and some sections $\beta_{l}$ of $\mathcal{H}^{k+i-n}\left(Y, \Omega_{Y}^{k+j-n}\right)$. Then, since the Newton polynomials, and also their Künneth components, are $d$-closed, we can write

$$
0=d \tilde{\gamma}_{n-i, n-j}^{k}(\mathcal{E})=\sum_{l}\left(d_{X} \alpha_{l} \otimes \beta_{l}+\alpha_{l} \otimes d_{Y} \beta_{l}\right) .
$$

Now, since $X$ is a smooth projective variety, we have $d_{X} \alpha_{l}=0$, hence it follows that

$$
\sum_{l} \alpha_{l} \otimes d_{Y} \beta_{l}=0
$$
By recalling the definition of the map $f$, we can write $f(\sigma)=\sum_{l}\left\langle\sigma, \alpha_{l}\right\rangle \beta_{l}$,
where

$$
\left\langle\sigma, \alpha_{l}\right\rangle=\int_{X} \sigma \wedge \alpha_{l}
$$

is the Serre duality pairing. It follows, by (2.1), that $d(f(\sigma))=$ $=\sum_{l}\left\langle\sigma, \alpha_{l}\right\rangle d \beta_{l}=0$.

We note, in particular, that for $k=n-i$ we obtain a natural map

$$
f: H^{i}\left(X, \Omega_{X}^{j}\right) \longrightarrow H^{0}\left(Y, \Omega_{Y}^{j-i}\right),
$$

for any $i \leq j$. It follows that we can construct closed holomorphic $p$-forms on $Y$ by starting with elements in $H^{i}\left(X, \Omega_{X}^{i+p}\right)$, for any $i \geq 0$. 
We remark that a similar construction is used in $[\mathrm{KM}]$ to define holomorphic symplectic structures on certain moduli spaces of sheaves on $X$, in some cases when $X$ does not possess any non-zero holomorphic 2-forms.

A more elementary construction of closed forms on moduli spaces of stable sheaves (valid only on the smooth locus of the moduli space) was given in [B3].

As an application, we will now show how these results can be applied in order to construct closed differential forms on Hilbert schemes of points.

Let $X$ be a smooth projective variety and let us denote by $X^{[d]}=$ $=\operatorname{Hilb}^{d}(X)$ the Hilbert scheme parametrizing 0-dimensional subschemes of $X$ of length $d . X^{[d]}$ is a projective scheme; it is smooth if $d \leq 3$ or $\operatorname{dim} X \leq 2$.

For any 0-dimensional subscheme $Z \in X^{[d]}$, let us denote by $\mathcal{I}_{Z}$ its sheaf of ideals: we have the standard exact sequence

$$
0 \rightarrow \mathcal{I}_{Z} \rightarrow \mathcal{O}_{X} \rightarrow \mathcal{O}_{Z} \rightarrow 0 .
$$

The Hilbert scheme $X^{[d]}$ can then be identified with the moduli space $\mathcal{M}$ parametrizing ideal sheaves $\mathcal{I}_{Z}$ of colength $d$. If $Z \in X^{[d]}$, it is well known that the tangent space $T_{Z} X^{[d]}$ is given by

$$
T_{Z} X^{[d]} \cong \operatorname{Hom}\left(\mathcal{I}_{Z}, \mathcal{O}_{Z}\right) .
$$

By applying the functor $\operatorname{Hom}\left(\cdot, \mathcal{O}_{Z}\right)$ to the exact sequence (2.3) and observing that $\operatorname{Ext}^{1}\left(\mathcal{O}_{X}, \mathcal{O}_{Z}\right) \cong H^{1}\left(X, \mathcal{O}_{Z}\right)=0$ and $\operatorname{Hom}\left(\mathcal{O}_{X}, \mathcal{O}_{Z}\right) \cong \operatorname{Hom}\left(\mathcal{O}_{Z}, \mathcal{O}_{Z}\right)$, since any $\mathcal{O}_{X}$-linear homomorphism from $\mathcal{O}_{X}$ to $\mathcal{O}_{Z}$ vanishes on $\mathcal{I}_{Z}$, we obtain a canonical isomorphism

$$
\operatorname{Hom}\left(\mathcal{I}_{Z}, \mathcal{O}_{Z}\right) \cong \operatorname{Ext}^{1}\left(\mathcal{O}_{Z}, \mathcal{O}_{Z}\right)
$$

The identification of $X^{[d]}$ with a moduli space of sheaves on $X$ allows us to apply, to the Hilbert scheme of points of $X$, the preceding results about the construction of closed differential forms. It follows that, given a $p$-form $\sigma \in H^{0}\left(X, \Omega_{X}^{p}\right)$, there is a naturally defined closed $p$-form $\tilde{\sigma}$ on $X^{[d]}$. At a point $Z \in X^{[d]}$ whose support consists of $d$ distinct points $P_{1}, \ldots, P_{d}$ of $X$, the map

$$
\tilde{\sigma}(Z): T_{Z} X^{[d]} \times \cdots \times T_{Z} X^{[d]} \rightarrow k
$$

can be easily described. First of all, note that such a $Z$ is a smooth point of $X^{[d]}$, and we have

$$
T_{Z} X^{[d]}=\bigoplus_{i=1}^{d} T_{P_{i}} X
$$


For any point $P_{i}$ in the support of $Z$ we have the map

$$
\sigma\left(P_{i}\right): T_{P_{i}} X \times \cdots \times T_{P_{i}} X \rightarrow k .
$$

Then the map $\tilde{\sigma}(Z)$ coincides (possibly up to a non-zero constant factor) with the product map $\prod_{i=1}^{d} \sigma\left(P_{i}\right)$.

Note that this explicit description allows us to define the $p$-form $\tilde{\sigma}$ only on the open subset $U$ of $X^{[d]}$ parametrizing subschemes of $X$ whose support consists of $d$ distinct points. What our method shows is that the $p$-form $\tilde{\sigma}$ thus defined on $U$ actually extends as a closed differential form on the whole of $X^{[d]}$ (see also [Be] for the construction of closed 2-forms on $X^{[d]}$, when $X$ is a $\mathrm{K} 3$ surface).

Acknowledgments. We would like to thank the referee for some valuable comments and suggestions.

\section{REFERENCES}

[A] I. V. ARTamkin, On deformation of sheaves, Math. USSR Izvestiya, 32 (1989), pp. 663-668.

[Be] A. BEAUVILLE, Variétés kählériennes dont la première classe de Chern est nulle, J. Diff. Geom., 18 (1983), pp. 755-782.

[B1] F. BotTaCin, Poisson structures on moduli spaces of sheaves over Poisson surfaces, Invent. Math., 121 (1995), pp. 421-436.

[B2] F. BotTacin, Poisson structures on Hilbert schemes of points of a surface and integrable systems, Manuscripta Math., 97 (1998), pp. 517-527.

[B3] F. BotTaCin, Closed differential forms on moduli spaces of sheaves, Rend. Mat. Univ. Roma, 28 (2008), pp. 1-24.

[EGA4] A. Grothendieck, Étude locale des schémas et des morphismes de schémas, Publ. Math. IHES, 1964-67.

[HL] D. HuYBrechtS - M. LeHn, The geometry of moduli spaces of sheaves, Aspects of Mathematics, Vol. E31, Vieweg, 1997.

[K] S. KoBAYASHI, Simple vector bundles over symplectic Kähler manifolds, Proc. Japan Acad., 62 (1986), pp. 21-24.

[KM] S. KUZNETSOV - D. MarkUShevich, Symplectic structures on moduli spaces of sheaves via the Atiyah class, math.AG/0703264 (2007).

[Ma] M. Maruyama, Moduli of stable sheaves II, J. Math. Kyoto Univ., 18 (1978), pp. 557-614.

[Mu1] S. MukaI, Symplectic structure of the moduli space of sheaves on an abelian or K3 surface, Invent. Math., 77 (1984), pp. 101-116.

[Mu2] S. MukaI, Moduli of vector bundles on K3 surfaces and symplectic manifolds, Sugaku Expositions, 1 (1988), pp. 139-174.

[R] Z. RAN, On the local geometry of moduli spaces of locally free sheaves, 
Moduli of vector bundles (Sanda, 1994; Kyoto, 1994), pp. 213-219, Lecture Notes in Pure and Appl. Math., 179 (Dekker, New York, 1996).

[S] C. Simpson, Moduli of representations of the fundamental group of a smooth projective variety I, Publ. Math. I.H.E.S., 79 (1994), pp. 47-129.

[Ty] A. N. TYURIN, Symplectic structures on the varieties of moduli of vector bundles on algebraic surfaces with $p_{g}>0$, Math. USSR Izvestiya, 33 (1989), pp. 139-177.

Manoscritto pervenuto in redazione il 21 gennaio 2008. 
